\title{
Robust stability for sampled-data control systems
}

\author{
Dennis S. BERNSTEIN \\ Harris Corporation, Government Aerospace Systems Division, MS 22/4842, Melbourne, FL 32902, U.S.A.
}

\section{C.V. HOLLOT}

Department of Electrical and Computer Engineering, Unlversity of Massachusetts, Amherst, MA 01003, U.S.A.

Received 28 January 1989

Revised 26 May 1989

\begin{abstract}
We consider the robust stability of a continuous-time system under computer control. The uncertainty is modeled as additive perturbations to the matrices in a continuous-time state space description of the plant. Our methods exploit the resulting exponential-like uncertainty structure in the sampled-data control system and we develop sufficient conditions for such a system to be robustly stable.
\end{abstract}

Keywords: Robust stability; sampled-data control; exponential-like uncertainty.

\section{Introduction}

A sampled-data control system consists of a continuous-time plant under computer control. Generally speaking, if the matrices in a state space description of a continuous-time plant are uncertain, then the resulting closed-loop, discrete-time system possesses an exponential-like uncertainty structure. This is true even if the continuous-time plant has linear uncertainty. Existing methods, such as [5-8], are inadequate in analyzing such uncertain discrete-time systems since they do not directly handle these exponential-like structures. Notable exceptions include the conic sector approach in [11], and the stochastic parameter formulation in [12]. Indeed, the present paper was motivated by the approach of [12] which, as shown in [2], can be reinterpreted to yield conditions for deterministic robust stability. Our objective is thus to develop a robust stability test which exploits the specific nonlinear uncertainty structures arising in sampled-data control systems.

In the sequel, the following notation will be used. For $X \in R^{n \times n}, X^{\prime}$ denotes the transpose of $X$, while $X>0(X \geqslant 0)$ means that $X$ is positive definite (positive semi-definite). The spectral radius of $X$ is given by $\rho(X)$. Let $\otimes, \oplus$ and 'vec' denote the Kronecker product, Kronecker sum and column stacking operators respectively; see [4]. In addition we shall use ' $\mathrm{vec}^{-1}$ ' to denote the operation of forming a (usually square) matrix from a column vector.

\section{Problem formulation}

In this section we state the robust analysis problem for sampled-data control systems using static feedback. To begin, consider the $n$-dimensional continuous-time plant

$$
\dot{x}(t)=(A+\Delta A) x(t)+(B+\Delta B) u(t), \quad y(t)=C x(t),
$$

where $A \in R^{n \times n}, B \in R^{n \times m}$ and $C \in R^{q \times n}$ denote nominal state space matrices and where $\Delta A$ and $\Delta B$ 
represent perturbations in $A$ and $B$ respectively. The pair of suitably dimensioned matrices $(\Delta A, \Delta B)$ belongs to an uncertainty set $U$ given by

$$
U=\left\{(\Delta A, \Delta B): \Delta A=\sum_{i=1}^{p} \sigma_{i} A_{i} \text { and } \Delta B=\sum_{i=1}^{p} \sigma_{i} B_{i}, \sum_{i=1}^{p} \sigma_{i}^{2} \leqslant 1\right\}
$$

where $A_{i}$ and $B_{i}$ reflect the 'structure' of the uncertainty and where $\sigma_{i}$ is an uncertain real parameter. Note that an uncertain parameter $\sigma_{i}$ may appear in both $\Delta A$ and $\Delta B$, and it's possible to have $A_{i}=0$ and $B_{i} \neq 0$ or vice-versa.

Now, consider a sampled-data system with a sampling period of $h$ seconds. We assume perfect synchronization between the A/D (sampler) and the (D/A) (zero-order hold) and ignore finite word-length effects and computational delays. We also assume that a static control law

$$
u(k h)=K y(k h), \quad k=0,1,2, \ldots,
$$

is implemented for some given gain $K \in R^{m \times q}$.

Our purpose is the analyze the robust stability of this closed-loop system, and to this end we consider its evolution at the sample instances $k h$ by forming the associated discrete-time system

$$
x(k+1)=\left[\mathrm{e}^{(A+\Delta A) h}+\int_{0}^{h} \mathrm{e}^{(A+\Delta A) \tau} \mathrm{d} \tau(B+\Delta B) K C\right] x(k) .
$$

In the above $x(k)$ denotes $x(k h)$; we have abused notation for the sake of conciseness. Given arbitrary $(\Delta A, \Delta B) \in U$, system (2.3) is discrete-time stable if all the eigenvalues of

$$
\mathrm{e}^{(A+\Delta A) h}+\int_{0}^{h} \mathrm{e}^{(A+\Delta A) \tau} \mathrm{d} \tau(B+\Delta B) K C
$$

lie within the open unit disk. Additionally, (2.3) is said to be robustly discrete-time stable if it is discrete-time stable for all $(\Delta A, \Delta B) \in U$.

\section{Main result}

We now develop a sufficient condition for the robust discrete-time stability of (2.3). This condition exploits the exponential structure of the uncertainty, i.e., rather than 'overbounding' the uncertainty with an additive model of the form

$$
x(k+1)=(\Phi+\Delta \Phi) x(k)
$$

our method treats the exponential structure of the nonlinearity in (2.3) more directly.

To show robust stability we will construct a parameter-independent (independent of the uncertain matrices $(\Delta A, \Delta B)$ ) quadratic Lyapunov function $V(x)$. Thus, let $P$ be some free, positive-definite symmetric matrix and consider the quadratic Lyapunov candidate

$$
V(x)=x^{\prime} P x, \quad x \in R^{n} \text {. }
$$

System (2.3) is robustly discrete-time stable if

$$
\begin{aligned}
\Delta V(\Delta A, \Delta B, P) \triangleq & {\left[\mathrm{e}^{(A+\Delta A) h}+\int_{0}^{h} \mathrm{e}^{(A+\Delta A) \tau} \mathrm{d} \tau(B+\Delta B) K C\right] P } \\
& \cdot\left[\mathrm{e}^{(A+\Delta A) h}+\int_{0}^{h} \mathrm{e}^{(A+\Delta A) \tau} \mathrm{d} \tau(B+\Delta B) K C\right]^{\prime}-P
\end{aligned}
$$

is negative definite for all $(\Delta A, \Delta B) \in U$. This follows since $x^{\prime} \Delta V x$ is the Lyapunov difference associated with $V=x^{\prime} P x$ and the adjoint of (2.3); see [1]. Recall that (2.3) has eigenvalues only in the open unit disk 
if and only if its adjoint system has eigenvalues only in the open unit disk. We use the Lyapunov difference for the adjoint system since some robust performance issues are related to such (covariance) equations; e.g., see [3] and [15].

A critical step in our development is to eliminate the integrals in (3.1) and express the uncertainty in terms of a single matrix exponential. Indeed, using the identity (see [13])

$$
\exp \left(\left[\begin{array}{cc}
(A+\Delta A) & (B+\Delta B) \\
0 & 0
\end{array}\right] h\right)=\left[\begin{array}{cc}
\mathrm{e}^{(A+\Delta A) h} & \int_{0}^{h} \mathrm{e}^{(A+\Delta A) \tau} \mathrm{d} \tau(B+\Delta B) \\
0 & I
\end{array}\right]
$$

(3.1) can be rewritten as

$$
\begin{aligned}
\Delta V(\Delta A, \Delta B, P)= & {\left[\begin{array}{ll}
I & 0
\end{array}\right] \exp \left(\left[\begin{array}{cc}
(A+\Delta A) & (B+\Delta B) \\
0 & 0
\end{array}\right] \cdot h\right)\left[\begin{array}{c}
I \\
K C
\end{array}\right] P\left[\begin{array}{ll}
I & \left.C^{\prime} K^{\prime}\right]
\end{array}\right.} \\
& \cdot \exp \left(\left[\begin{array}{cc}
(A+\Delta A) & (B+\Delta B) \\
0 & 0
\end{array}\right]^{\prime} h\right)\left[\begin{array}{l}
I \\
0
\end{array}\right]-P .
\end{aligned}
$$

Our next result provides a parameter-independent upper bound to $\Delta V$. To give this bound, let $\alpha>0$ be given and define

$$
\overline{A_{\alpha}} \triangleq\left(\left[\begin{array}{cc}
A & B \\
0 & 0
\end{array}\right]+\frac{1}{2} \alpha I\right) \oplus\left(\left[\begin{array}{cc}
A & B \\
0 & 0
\end{array}\right]+\frac{1}{2} \alpha I\right)+\frac{1}{\alpha} \sum_{i=1}^{p}\left[\begin{array}{cc}
A_{i} & B_{i} \\
0 & 0
\end{array}\right] \otimes\left[\begin{array}{cc}
A_{i} & B_{i} \\
0 & 0
\end{array}\right]
$$

and

$$
\Pi_{P} \triangleq\left[\begin{array}{c}
I \\
K C
\end{array}\right] P\left[\begin{array}{ll}
I & C^{\prime} K^{\prime}
\end{array}\right]
$$

Lemma 3.1 (see Appendix for proof). If $P \in R^{n \times n}$ is positive definite and symmetric and $\alpha>0$, then

$$
\begin{aligned}
\Delta V(\Delta A, \Delta B, P)= & {\left[\begin{array}{ll}
I & 0
\end{array}\right] \exp \left(\left[\begin{array}{cc}
(A+\Delta A) & (B+\Delta B) \\
0 & 0
\end{array}\right] h\right)\left[\begin{array}{c}
I \\
K C
\end{array}\right] P\left[\begin{array}{ll}
I & C^{\prime} K^{\prime}
\end{array}\right] } \\
& \cdot \exp \left(\left[\begin{array}{cc}
(A+\Delta A) & (B+\Delta B) \\
0 & 0
\end{array}\right]^{\prime} h\right)\left[\begin{array}{l}
I \\
0
\end{array}\right]-P \\
\leqslant & {\left[\begin{array}{ll}
I & 0
\end{array}\right] \operatorname{vec}^{-1}\left[\mathrm{e}^{\bar{A}_{\alpha} h} \operatorname{vec}\left(\Pi_{P}\right)\right]\left[\begin{array}{l}
I \\
0
\end{array}\right]-P }
\end{aligned}
$$

for all $(\Delta A, \Delta B) \in U$.

Now, using the right-hand side of (3.6), formally set

$$
\left[\begin{array}{ll}
I & 0
\end{array}\right] \operatorname{vec}^{-1}\left[\mathrm{e}^{\bar{T}_{\alpha} h} \operatorname{vec}\left(\Pi_{P}\right)\right]\left[\begin{array}{l}
I \\
0
\end{array}\right]-P=-I
$$

which is equivalent to

$$
\left(M_{\alpha}-I\right) \operatorname{vec}(P)=-\operatorname{vec}(I)
$$

where

$$
M_{\alpha} \triangleq\left(\left[\begin{array}{ll}
I & 0
\end{array}\right] \otimes\left[\begin{array}{ll}
I & 0
\end{array}\right]\right) \mathrm{e}^{\bar{A}_{\alpha} h}\left(\left[\begin{array}{c}
I \\
K C
\end{array}\right] \otimes\left[\begin{array}{c}
I \\
K C
\end{array}\right]\right)
$$

When does (3.8) have a positive-definite solution? Our next lemma gives a sufficient condition. 
Lemma 3.2 (see Appendix for proof). If there exists an $\alpha>0$ such that

$$
\rho\left(M_{\alpha}\right)<1,
$$

then (3.8) has a positive-definite solution $P$.

Now, assume (3.10) holds for some $\alpha>0$. From Lemma 3.2, equation (3.7) has a positive-definite solution $P$; hence, using (3.6) and (3.7) it follows that

$$
\Delta V(\Delta A, \Delta B, P) \leqslant-I
$$

for all $(\Delta A, \Delta B) \in U$. We have thus proven the following main result.

Theorem 3.1 If there exists an $\alpha>0$ such that

$$
\rho\left(M_{\alpha}\right)<1,
$$

then the sampled-data system (2.3) is robustly discrete-time stable.

It is important to note that the condition of Theorem 3.1, $\rho\left(M_{\alpha}\right)<1$, is always satisfied for sufficiently small $\alpha>0$ if the nominal system is stable and there is no uncertainty; i.e., $\Delta A=0$ and $\Delta B=0$. Indeed, taking $\alpha \simeq 0$, a straightforward manipulation using (3.2), (3.4), (3.9) and identities (A.1)-(A.4) in the Appendix gives

$$
M_{\alpha}=\left(\mathrm{e}^{A h}+\int_{0}^{h} \mathrm{e}^{A \tau} \mathrm{d} \tau B K C\right) \otimes\left(\mathrm{e}^{A h}+\int_{0}^{h} \mathrm{e}^{A \tau} \mathrm{d} \tau B K C\right) .
$$

Since the nominal system is stable, all the eigenvalues of

$$
\mathrm{e}^{A h}+\int_{0}^{h} \mathrm{e}^{A \tau} \mathrm{d} \tau B K C
$$

lie within the unit disk. This implies, together with (3.12) and the fact that the eigenvalues of a Kronecker product of two matrices are the products of the eigenvalues of these two matrices (see [4]), that all the eigenvalues of $M_{\alpha}$ lie within the unit disk. Thus, $\rho\left(M_{\alpha}\right)<1$.

Finally, we remark that a dual result holds when one allows uncertainty in $C$; i.e., $C \rightarrow C+\Delta C$.

\section{Example}

In Soroka and Shaked [10], the robustness of a continuous-time system under 'cheap' LQ regulation is studied. This uncertain closed-loop system is described by

$$
\begin{aligned}
& \dot{x}(t)=\left[\begin{array}{rr}
-1 & 0 \\
0 & -2
\end{array}\right] x(t)+\left[\begin{array}{c}
1-\sigma_{1} \\
1
\end{array}\right] u(t), \quad \sigma_{1}>0, \\
& z(t)=\left[\begin{array}{ll}
-1 & 1
\end{array}\right] x(t), \quad u(t)=L_{r} x(t),
\end{aligned}
$$

where the $L Q$ regulator gains are given by

$$
L_{r}=\left[\begin{array}{lll}
1+q_{r}-\sqrt{5+2 q_{r}} & 2 \sqrt{5+2 q_{r}}-q_{r}-4
\end{array}\right], \quad q_{r}=\sqrt{4+1 / r},
$$

with $r>0$ being the control weighting in the quadratic performance index

$$
\int_{0}^{\infty}\left[z^{2}(t)+r u^{2}(t)\right] \mathrm{d} t
$$




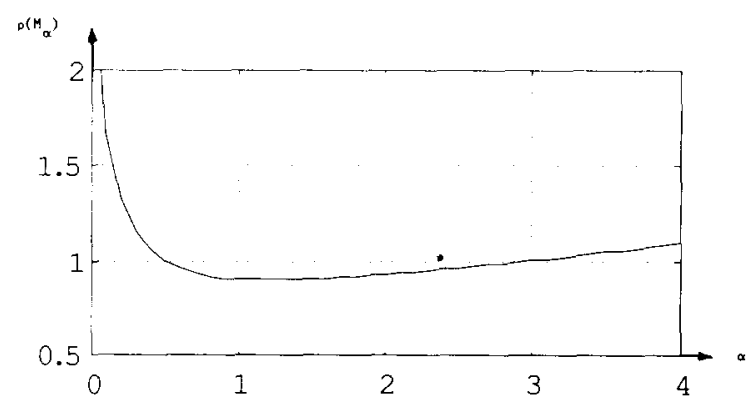

Fig. 1. If $\rho\left(M_{\alpha}\right)<1$ for some $\alpha>0$, then the sampled-data control system (2.3) is robustly discrete-time stable; see Theorem 3.1. In the above we plot $\rho\left(M_{\alpha}\right)$ versus $\alpha$ for the system matrices in (4.2)-(4.3) with $h=0.1, r=0.008$ and $\bar{\sigma}=0.5$. Since $\rho\left(M_{\alpha}\right)<1$ for all $\alpha \in(0.6,2.8)$, this system is robustly discrete-time stable.

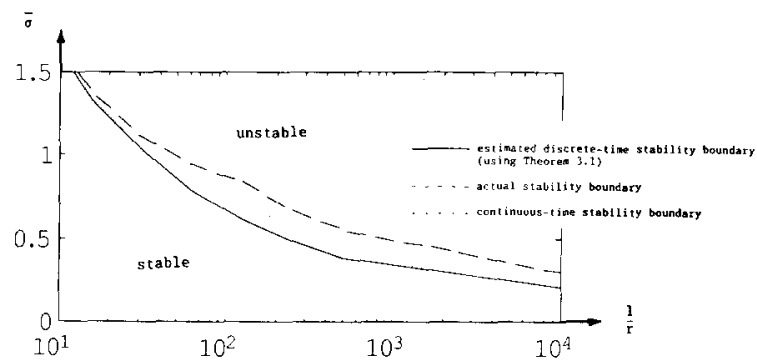

Fig. 2. The results of Theorem 3.1 provide a means for estimating stability boundaries. In this figure we consider the closedloop system in (4.3)-(4.4) and plot this estimate against the actual stability boundary as determined by (4.5). The agreement is quite good. The continuous-time stability boundary is plotted for completeness.

Soroka and Shaked show that stability robustness over the range of parameters $\sigma_{1} \in\left[\begin{array}{lll}0 & \bar{\sigma}\end{array}\right], \bar{\sigma}>0$, decreases $(\bar{\sigma} \rightarrow 0)$ as the control becomes cheaper $(r \rightarrow 0)$. In terms of the system formulation $(\Sigma)$ we have

$$
\begin{aligned}
& A=\left[\begin{array}{rr}
-1 & 0 \\
0 & -2
\end{array}\right] \quad B=\left[\begin{array}{c}
1-\frac{1}{2} \bar{\sigma} \\
1
\end{array}\right], \quad C=I, \\
& \Delta A=0, \quad \Delta B=\sigma_{1} B_{1}, \quad B_{1}=\left[\begin{array}{c}
\frac{1}{2} \bar{\sigma} \\
0
\end{array}\right], \quad \sigma_{1}^{2} \leqslant 1 .
\end{aligned}
$$

Now, suppose this LQ regulator gain is to be implemented in a computer. What is the robustness of this sampled-data control system? We will use the condition in Theorem 3.1 to answer this question. First, however, we must translate the continuous-time $L Q$ gain to one suitable for sampled-data control. Indeed, the nominal discrete-time system is unstable if we implement gain $K=L_{r}$ in the computer. Following [1, pp. 189-191], we take

$$
K=-L_{r}\left[I+\frac{1}{2}\left(A-B L_{r}\right) h\right] .
$$

For a given sampling period $h$, control weighting $r$ and uncertainty bound $\bar{\sigma}$, we are now in a position to determine if $\rho\left(M_{\alpha}\right)<1$ for some $\sigma>0$. For example, with $h=0.1, r=0.008$ and $\bar{\sigma}=0.5, \rho\left(M_{\alpha}\right)<1$ for all $\alpha \in(0.6,2.8)$; see Figure 1. Hence, the sampled-data system is stable for $\bar{\sigma}=0.5$. Also, for $h=0.1$ we determine, for various $r$, the largest $\bar{\sigma}$ for which $\rho\left(M_{\alpha}\right)<1$ for some $\alpha>0$. We compare these results to the actual discrete-time stability boundary and to the continuous-time $L Q$ solution in [10]; see Figure 2 . To compute the actual discrete-time stability boundary, we assume time-invariant uncertainty $\sigma_{1}$ and find the largest $\bar{\sigma}$ for which

$$
\rho\left(\mathrm{e}^{A h}+\int_{0}^{h} \mathrm{e}^{A \tau} \mathrm{d} \tau\left[\begin{array}{c}
1-\sigma_{1} \\
1
\end{array}\right] K\right)<1 .
$$

for all $\left|\sigma_{1}\right| \leqslant \bar{\sigma}$. Finally, we note that the actual discrete time stability region is larger than its continuous-time counterpart.

\section{Appendix: Proof of Lemmas 3.1 and 3.2}

To prove Lemmas 3.1 and 3.2 we first need some identities and observations; see [4] for details. 
Identities. If $X \in R^{r \times r}$ and $Y \in R^{x \times s}$, then by definition the Kronecker sum is given by

$X \oplus Y=X \otimes I+I \otimes Y$.

Next, if the indicated products exist, then

$$
\begin{aligned}
& \operatorname{vec}(X Y Z)=\left(Z^{\prime} \otimes X\right) \operatorname{vec}(Y), \\
& (X \otimes Y)(Z \otimes W)=(X Z) \otimes(Y W) .
\end{aligned}
$$

Finally, if $X$ is square, then

$$
\mathrm{e}^{X \oplus X}=\mathrm{e}^{X} \otimes \mathrm{e}^{X} .
$$

Observation 1. Given arbitrary $(\Delta A, \Delta B) \in U$, the solution to the matrix differential equation

$$
\dot{Y}(t)=\left[\begin{array}{cc}
(A+\Delta A) & (B+\Delta B) \\
0 & 0
\end{array}\right] Y(t)+Y(t)\left[\begin{array}{cc}
(A+\Delta A) & (B+\Delta B) \\
0 & 0
\end{array}\right]^{\prime}, \quad t \geqslant 0, \quad Y(0)=Y_{0},
$$

is

$$
Y(t)=\exp \left(\left[\begin{array}{cc}
(A+\Delta A) & (B+\Delta B) \\
0 & 0
\end{array}\right] t\right) Y_{0} \exp \left(\left[\begin{array}{cc}
(A+\Delta A) & (B+\Delta B) \\
0 & 0
\end{array}\right]^{\prime} t\right)
$$

This is a well-known result.

Observation 2. Let $\alpha>0$; then the solution of the matrix differential equation

$$
\begin{aligned}
\dot{\bar{Y}}(t)= & \left(\left[\begin{array}{cc}
A & B \\
0 & 0
\end{array}\right]+\frac{1}{2} \alpha I\right) \bar{Y}(t)+\bar{Y}(t)\left(\left[\begin{array}{cc}
A & B \\
0 & 0
\end{array}\right]^{\prime}+\frac{1}{2} \alpha I\right) \\
& +\frac{1}{\alpha} \sum_{i=1}^{p}\left[\begin{array}{cc}
A_{i} & B_{i} \\
0 & 0
\end{array}\right] \bar{Y}(t)\left[\begin{array}{cc}
A_{i} & B_{i} \\
0 & 0
\end{array}\right]^{\prime}, \quad t \geqslant 0, \quad \bar{Y}(0)=\bar{Y}_{0},
\end{aligned}
$$

is

$$
\bar{Y}(t)=\operatorname{vec}^{-1}\left[\mathrm{e}^{\bar{A}_{\alpha} t} \operatorname{vec}\left(\bar{Y}_{0}\right)\right]
$$

To show (A.8), apply the 'vec' operation to both sides of (A.7) and use identities (A.1) and (A.2) to get

$$
\operatorname{vec}(\dot{\bar{Y}}(t))=\left\{\left(\left[\begin{array}{cc}
A & B \\
0 & 0
\end{array}\right]+\frac{1}{2} \alpha I\right) \oplus\left(\left[\begin{array}{cc}
A & B \\
0 & 0
\end{array}\right]+\frac{1}{2} \alpha I\right)+\frac{1}{\alpha} \sum_{i=1}^{p}\left(\left[\begin{array}{cc}
A_{i} & B_{i} \\
0 & 0
\end{array}\right] \otimes\left[\begin{array}{cc}
A_{i} & B_{i} \\
0 & 0
\end{array}\right]\right)\right\} \operatorname{vec}(\bar{Y}(t)) .
$$

The solution to the above is $\operatorname{vec}(\bar{Y}(t))=\left[\mathrm{e}^{\bar{A}_{\alpha} t} \operatorname{vec}\left(\bar{Y}_{0}\right)\right]$, from which (A.8) follows.

Observation 3. If $\alpha>0$ and if $\bar{Y}_{0}$ is positive semi-definite, then the solution in (A.8) satisfies

$$
\bar{Y}(t)=\operatorname{vec}^{-1}\left[\mathrm{e}^{\bar{A}_{\alpha} t} \operatorname{vec}\left(\bar{Y}_{0}\right)\right] \geqslant 0, \quad t \geqslant 0 .
$$

To prove this observation, let $S, N \in R^{r \times r}$ with $N$ positive semi-definite. From (A.2),

$$
(S \otimes S) \operatorname{vec}(N)=\operatorname{vec}\left(S N S^{\prime}\right)
$$

which implies

$$
\operatorname{vec}^{-1}[(S \otimes S) \operatorname{vec}(N)]=S N S^{\prime} \geqslant 0 .
$$


Using (A.3),

$$
(S \otimes S)^{i}=\left(S^{i} \otimes S^{i}\right)
$$

so that

$$
\begin{aligned}
\operatorname{vec}^{-1}\left[\mathrm{e}^{S \otimes S} \operatorname{vec}(N)\right] & =\operatorname{vec}^{-1}\left[\sum_{i=0}^{\infty}(i !)^{-1}(S \otimes S)^{i} \operatorname{vec}(N)\right] \\
& =\operatorname{vec}^{-1}\left[\sum_{i=0}^{\infty}(i !)^{-1}\left(S^{i} \otimes S^{i}\right) \operatorname{vec}(N)\right] \\
& =\sum_{i=0}^{\infty} \operatorname{vec}^{-1}\left[(i !)^{-1}\left(S^{i} \otimes S^{i}\right) \operatorname{vec}(N)\right]=\sum_{i=0}^{\infty}(i !)^{-1} S^{i} N S^{i \prime} \geqslant 0
\end{aligned}
$$

Furthermore, from (A.4) and (A.10),

$$
\operatorname{vec}^{-1}\left[\mathrm{e}^{S \oplus S} \operatorname{vec}(N)\right]=\operatorname{vec}^{-1}\left[\left(\mathrm{e}^{S} \otimes \mathrm{e}^{S}\right) \operatorname{vec}(N)\right]=\mathrm{e}^{S} N \mathrm{e}^{S \prime} \geqslant 0 .
$$

Now, using the exponential product formula (see [14, pg. 97]) we write

$$
\mathrm{e}^{\overline{A_{\alpha}} t}=\lim _{j \rightarrow \infty}\left\{\exp \left[\frac{1}{j}\left(\tilde{A_{\alpha}} \oplus \tilde{A_{\alpha}}\right) t\right] \prod_{i=1}^{p} \exp \left[\frac{1}{\alpha j}\left(\tilde{A_{i}} \otimes \tilde{A_{i}}\right) t\right]\right\}^{j}
$$

where

$$
\tilde{A_{\alpha}} \triangleq\left[\begin{array}{cc}
A & B \\
0 & 0
\end{array}\right]+\frac{1}{2} \alpha I, \quad \tilde{A_{i}} \triangleq\left[\begin{array}{cc}
A_{i} & B_{i} \\
0 & 0
\end{array}\right] .
$$

Consequently,

$$
\begin{aligned}
& \operatorname{vec}^{-1}\left[\mathrm{e}^{\bar{A}_{\alpha} t} \operatorname{vec}\left(\bar{Y}_{0}\right)\right]=\operatorname{vec}^{-1}\left\{\lim _{j \rightarrow \infty}\left\{\exp \left[\frac{1}{j}\left(\tilde{A}_{\alpha} \oplus \tilde{A}_{\alpha}\right) t\right] \prod_{i=1}^{p} \exp \left[\frac{1}{\alpha j}\left(\tilde{A}_{i} \otimes \tilde{A}_{i}\right) t\right]\right\}^{j} \operatorname{vec}\left(\bar{Y}_{0}\right)\right\} \\
& =\lim _{j \rightarrow \infty}\left\{\operatorname{vec}^{-1}\left\{\left\{\exp \left[\frac{1}{j}\left(\tilde{A_{\alpha}} \oplus \tilde{A_{\alpha}}\right) t\right] \prod_{i=1}^{p} \exp \left[\frac{1}{\alpha j}\left(\tilde{A_{i}} \otimes \tilde{A_{i}}\right) t\right]\right\}^{j} \operatorname{vec}\left(\overline{Y_{0}}\right)\right\}\right\} \text {. }
\end{aligned}
$$

We will now show that the expression in the limit brackets in (A.16) is positive semi-definite for all positive integers $j$. Indeed, this is sufficient to prove that (A.9) holds. For simplicity take $j=p=1$ and let $\tilde{N}$ satisfy

$$
\operatorname{vec}(\tilde{N})=\exp \left[\frac{1}{\alpha}\left(\tilde{A}_{i} \otimes \tilde{A_{i}}\right) t\right] \operatorname{vec}\left(\bar{Y}_{0}\right)
$$

or equivalently

$$
\tilde{N}=\operatorname{vec}^{-1}\left\{\exp \left[\frac{1}{\alpha}\left(\tilde{A_{i}} \otimes \tilde{A_{i}}\right) t\right] \operatorname{vec}\left(\bar{Y}_{0}\right)\right\} .
$$

Since $\bar{Y}_{0}$ is assumed positive semi-definite, then, from (A.12), $\tilde{N}$ is positive semi-definite. From (A.13), (A.17) and this fact, the expression in the limit brackets of (A.16) satisfies

$$
\operatorname{vec}^{-1}\left\{\exp \left[\left(\tilde{A}_{\alpha} \oplus \tilde{A_{\alpha}}\right) t\right] \exp \left[\frac{1}{\alpha}\left(\tilde{A_{i}} \otimes \tilde{A_{i}}\right) t\right] \operatorname{vec}\left(\overline{Y_{0}}\right)\right\}=\operatorname{vec}^{-1}\left\{\exp \left[\left(\tilde{A_{\alpha}} \oplus \tilde{A_{\alpha}}\right) t\right] \operatorname{vec}(\tilde{N})\right\} \geqslant 0
$$


A similar argument, using (A.12) and (A.13) alternately, shows that the expression in the limit brackets of (A.16) is positive semi-definite for arbitrary positive integers $j$ and $p$. This completes the proof of Observation 3.

Proof of Lemma 3.1. Assume $P$ positive definite and $\alpha>0$. Furthermore, consider the matrix differential equations in (A.5) and (A.7) with

$$
Y_{0}=\bar{Y}_{0}=\Pi_{P}
$$

where $\Pi_{P}$ is given in (3.5). Subtracting (A.5) and (A.7) gives

$$
\begin{aligned}
\dot{\bar{Y}}(t)-\dot{Y}(t)= & {\left[\begin{array}{cc}
(A+\Delta A) & (B+\Delta B) \\
0 & 0
\end{array}\right](\bar{Y}(t)-Y(t)) } \\
& +(\bar{Y}(t)-Y(t))\left[\begin{array}{cc}
(A+\Delta A) & (B+\Delta B) \\
0 & 0
\end{array}\right]^{\prime}+\Psi_{\alpha}(t), \quad t \geqslant 0, \quad \bar{Y}(0)-Y(0)=0,
\end{aligned}
$$

where

$$
\begin{aligned}
\Psi_{\alpha}(t) \triangleq & \tilde{A}_{\alpha} \bar{Y}(t)+\bar{Y}(t) \tilde{A}_{\alpha}^{\prime}+\frac{1}{\alpha} \sum_{i=1}^{p} \tilde{A_{i}} \bar{Y}(t) \tilde{A}_{i}^{\prime} \\
& -\left(\left[\begin{array}{cc}
(A+\Delta A) & (B+\Delta B) \\
0 & 0
\end{array}\right] \bar{Y}(t)+\bar{Y}(t)\left[\begin{array}{cc}
(A+\Delta A) & (B+\Delta B) \\
0 & 0
\end{array}\right]^{\prime}\right)
\end{aligned}
$$

and where $\tilde{A}_{\alpha}$ and $\tilde{A}_{i}$ are defined in (A.15).

Claim 1: $\Psi_{\alpha}(t)$ is positive semi-definite for all $t \geqslant 0$.

Proof of Claim 1: From (A.15) and (A.21),

$$
\begin{aligned}
& \Psi_{\alpha}(t)=\left(\left[\begin{array}{cc}
A & B \\
0 & 0
\end{array}\right]+\frac{1}{2} \alpha I\right) \bar{Y}(t)\left(\left[\begin{array}{cc}
A & B \\
0 & 0
\end{array}\right]^{\prime}+\frac{1}{2} \alpha I\right) \\
& -\left(\left[\begin{array}{cc}
(A+\Delta A) & (B+\Delta B) \\
0 & 0
\end{array}\right] \bar{Y}(t)+\bar{Y}(t)\left[\begin{array}{cc}
(A+\Delta A) & (B+\Delta B) \\
0 & 0
\end{array}\right]^{\prime}\right)+\frac{1}{\alpha} \sum_{i=1}^{p} \tilde{A_{i}} \bar{Y}(t) \tilde{A}_{i}^{\prime} \\
& =\alpha \bar{Y}(t)-\left(\left[\begin{array}{cc}
\Delta A & \Delta B \\
0 & 0
\end{array}\right] \bar{Y}(t)+\bar{Y}(t)\left[\begin{array}{cc}
\Delta A & \Delta B \\
0 & 0
\end{array}\right]^{\prime}\right)+\frac{1}{\alpha} \sum_{i=1}^{p} \tilde{A}_{i} \bar{Y}(t) \tilde{A}_{i}^{\prime} \\
& \geqslant \alpha \sum_{i=1}^{p} \sigma_{i}^{2} \bar{Y}(t)-\sum_{i=1}^{p} \sigma_{i}\left(\tilde{A_{i}} \bar{Y}(t)+\bar{Y}(t) \tilde{A}_{i}^{\prime}\right)+\frac{1}{\alpha} \sum_{i=1}^{p} \tilde{A_{i}} \bar{Y}(t) \tilde{A}_{i}^{\prime} \\
& =\sum_{i=1}^{p}\left[\sqrt{\alpha} \sigma_{i} I-\frac{1}{\sqrt{\alpha}} \tilde{A_{i}}\right] \bar{Y}(t)\left[\sqrt{\alpha} \sigma_{i} I-\frac{1}{\sqrt{\alpha}} \tilde{A_{i}}\right]^{\prime} \text {. }
\end{aligned}
$$

From Observation 3, $\bar{Y}(t)$ is positive semi-definite for all $t \geqslant 0$. It thus follows from (A.22) that $\Psi_{\alpha}(t)$ is likewise positive semi-definite for all $t \geqslant 0$. This proves the claim.

Now, since $\bar{Y}(0)-Y(0)=0$, the solution to (A.20) is

$$
\begin{aligned}
\bar{Y}(t)-Y(t)= & \int_{0}^{t} \exp \left[\left[\begin{array}{cc}
(A+\Delta A) & (B+\Delta B) \\
0 & 0
\end{array}\right](t-s)\right] \Psi_{\alpha}(s) \\
& \cdot \exp \left[\left[\begin{array}{cc}
(A+\Delta A) & (B+\Delta B) \\
0 & 0
\end{array}\right]^{\prime}(t-s)\right] \mathrm{d} s .
\end{aligned}
$$


From (A.23) and Claim 1 it follows that

$$
\bar{Y}(t)-Y(t) \geqslant 0, \quad t \geqslant 0 .
$$

Combining (A.6) and (A.8) with (A.24) gives

$$
\begin{aligned}
0 \leqslant & \bar{Y}(h)-Y(h) \\
= & \operatorname{vec}^{-1}\left[\mathrm{e}^{\bar{A}_{\alpha} h} \operatorname{vec}\left(\Pi_{P}\right)\right]-\exp \left(\left[\begin{array}{cc}
(A+\Delta A) & (B+\Delta B) \\
0 & 0
\end{array}\right] h\right) \Pi_{P} \\
& \cdot \exp \left(\left[\begin{array}{cc}
(A+\Delta A) & (B+\Delta B) \\
0 & 0
\end{array}\right]^{\prime} h\right)
\end{aligned}
$$

which implies that

$$
\begin{aligned}
& {\left[\begin{array}{ll}
I & 0
\end{array}\right] \exp \left(\left[\begin{array}{cc}
(A+\Delta A) & (B+\Delta B) \\
0 & 0
\end{array}\right] h\right) \Pi_{P} \exp \left(\left[\begin{array}{cc}
(A+\Delta A) & (B+\Delta B) \\
0 & 0
\end{array}\right] ' h\right)\left[\begin{array}{l}
I \\
0
\end{array}\right]-P} \\
& \quad \leqslant\left[\begin{array}{ll}
I & 0
\end{array}\right] \operatorname{vec}^{-1}\left[\mathrm{e}^{\bar{A}_{\alpha} h} \operatorname{vec}\left(\Pi_{P}\right)\right]\left[\begin{array}{l}
I \\
0
\end{array}\right]-P,
\end{aligned}
$$

which is the desired result. The proof of Lemma 3.1 is complete.

Proof of Lemma 3.2. Assume $\alpha>0$ such that (3.10) holds. We must show that (3.8) has a positive-definite solution $P$. Indeed, since $\rho\left(M_{\alpha}\right)<1$, it follows that $M_{\alpha}-I$ is invertible and (3.8) has a unique solution $\operatorname{vec}(P)=\left(I-M_{\alpha}\right)^{-1} \operatorname{vec}(I)$ or $P=\operatorname{vec}^{-1}\left[\left(I-M_{\alpha}\right)^{-1} \operatorname{vec}(I)\right]$.Now, it follows from [9, Theorem 6.7.1] that $\left(I-M_{\alpha}\right)^{-1}=\sum_{i=0}^{\infty} M_{\alpha}^{i}$ where $M_{\alpha}^{0} \triangleq I$. Consequently,

$$
P=\operatorname{vec}^{-1}\left[\sum_{i=0}^{\infty} M_{\alpha}^{i} \operatorname{vec}(I)\right]=\sum_{i=0}^{\infty} \operatorname{vec}^{-1}\left[M_{\alpha}^{i} \operatorname{vec}(I)\right] .
$$

Claim 2: If $i$ is a positive integer, then

$$
\operatorname{vec}^{-1}\left[M_{\alpha}^{i} \operatorname{vec}(I)\right] \geqslant 0 \text {. }
$$

Proof of Claim 2: The proof proceeds by induction. From the definition of $M_{\alpha}$ in (3.9) and identity (A.2), we have for $i=1$,

$$
\begin{aligned}
\operatorname{vec}^{-1}\left[M_{\alpha} \operatorname{vec}(I)\right] & =\operatorname{vec}^{-1}\left\{\left[\begin{array}{ll}
I & 0
\end{array}\right] \mathrm{e}^{\bar{A}_{\alpha} h}\left(\left[\begin{array}{c}
I \\
K C
\end{array}\right] \otimes\left[\begin{array}{c}
I \\
K C
\end{array}\right]\right) \operatorname{vec}(I)\right\} \\
& =\operatorname{vec}^{-1}\left\{\left[\begin{array}{ll}
I & 0
\end{array}\right] \mathrm{e}^{\bar{A}_{\alpha} h} \operatorname{vec}\left(\left[\begin{array}{c}
I \\
K C
\end{array}\right]\left[\begin{array}{ll}
I & C^{\prime} K^{\prime}
\end{array}\right]\right)\right\} \\
& =\left[\begin{array}{ll}
I & 0
\end{array}\right] \operatorname{vec}^{-1}\left\{\mathrm{e}^{\bar{A}_{\alpha} h} \operatorname{vec}\left(\left[\begin{array}{c}
I \\
K C
\end{array}\right]\left[\begin{array}{ll}
I & C^{\prime} K^{\prime}
\end{array}\right]\right)\right\}\left[\begin{array}{l}
I \\
0
\end{array}\right] .
\end{aligned}
$$

It follows from (A.9), (A.27) and Observation 3 with

$$
\bar{Y}(0)=\left[\begin{array}{c}
I \\
K C
\end{array}\right]\left[\begin{array}{ll}
I & C^{\prime} K^{\prime}
\end{array}\right], \quad t=h,
$$

that $\operatorname{vec}^{-1}\left[M_{\alpha} \operatorname{vec}(I)\right] \geqslant 0$. Now, for the induction step, assume $\operatorname{vec}^{-1}\left[M_{\alpha}^{i} \operatorname{vec}(I)\right] \geqslant 0$. Then

$$
\begin{aligned}
\operatorname{vec}^{-1}\left[M_{\alpha}^{i+1} \operatorname{vec}(I)\right] & =\operatorname{vec}^{-1}\left[M_{\alpha} M_{\alpha}^{i} \operatorname{vec}(i)\right] \\
& =\operatorname{vec}^{-1}\left\{M_{\alpha} \operatorname{vec}\left[\operatorname{vec}^{-1}\left(M_{\alpha}^{i} \operatorname{vec}(I)\right)\right]\right\} .
\end{aligned}
$$


From (A.28) and Observation 3 with

$$
\bar{Y}(0)=\operatorname{vec}^{-1}\left[M_{\alpha}^{i} \operatorname{vec}(I)\right], \quad t=h,
$$

it follows that $\operatorname{vec}^{-1}\left[M_{\alpha}^{i+1} \operatorname{vec}(I)\right] \geqslant 0$. This proves the lemma.

\section{Acknowledgement}

We thank Mr. Mujdat Arabacioglu for his assistance in preparing the example.

\section{References}

[1] K.J. Åstrom and B. Wittenmark, Computer Controlled Systems, Theory and Design (Prentice-Hall, Englewood Cliffs, NJ, 1984).

[2] D.S. Bernstein, Robust static and dynamic output-feedback stabilization: deterministic and stochastic perspectives, IEEE Trans. Automat. Control 32 (12) (1987) 1076-1084.

[3] D.S. Bernstein and W.M. Haddad, Robust stability and performance analysis for state space systems via quadratic Lyapunov bounds, Proc. IEEE Conf. Decision and Control, Austin, TX (1988).

[4] J.W. Brewer, Kronecker products and matrix calculus in system theory, IEEE Trans. Circuits and Systems 25 (9) (1978) $772-781$.

[5] M. Corless, Stabilization of uncertain linear systems, Proc. IFAC Workshop on Model Error Concepts and Compensation, Boston, MA (1985).

[6] C.V. Hollot and M. Arabacioglu, lth-step Lyapunov min-max controllers: Stabilizing discrete-time systems under real parameter variations, Proc. American Control Conference, Minneapolis, MN (1987) 496-501.

[7] M.E. Magana and S. Zak, Robust state feedback stabilization of discrete-time uncertain dynamical systems, IEEE Trans. Automat. Control 33 (9) (1988) 887-891.

[8] J. Manela, Deterministic Control of Uncertain Linear Discrete and Sampled-Data Systems, Ph.D. Dissertation, Univ. Calif., Berkeley, CA (1985).

[9] A.W. Naylor and G.R. Sell, Linear Operator Theory in Engineering and Science (Holt, Rinehart and Winston, New York, 1971).

[10] E. Soroka and U. Shaked, On the robustness of LQ regulators, IEEE Trans. Automat. Control 29 (7) 1984) $664-665$.

[11] P.M. Thompson, R.L. Dailey and J.C. Doyle, New conic sectors for sampled-data feedback systems, Systems Control Lett. 7 (1986) 395-404.

[12] A.R. Tiedemann and W.L. De Koning, The equivalent discrete-time optimal control problem for continuous-time systems with stochastic parameters, Internat. J. Control 40 (3) (1984) 449-466.

[13] C.F. Van Loan, Computing integrals involving the matrix exponential, IEEE Trans. Automat. Control 23 (3) (1978) $395-404$.

[14] V.S. Varadarajan, Lie Groups, Lie Algebras, and Their Representations (Springer-Verlag, New York, 1984).

[15] D.A. Wilson, Convolution and Hankel operator norms for linear systems, IEEE Trans. Automat. Control 34 (1) (1988) 94-97. 